\title{
Limit Cycles for the Class of $D$-Dimensional Polynomial Differential Systems
}

\author{
Zouhair Diab $^{1}$ and Amar Makhlouf ${ }^{2}$ \\ ${ }^{1}$ Department of Mathematics, University of Tébessa, 12002 Tébessa, Algeria \\ ${ }^{2}$ Department of Mathematics, Faculty of Sciences, UBM of Annaba, El Hadjar, 23000 Annaba, Algeria \\ Correspondence should be addressed to Zouhair Diab; diabzouhair@gmail.com
}

Received 8 November 2015; Revised 5 January 2016; Accepted 15 February 2016

Academic Editor: Hui-Shen Shen

Copyright (C) 2016 Z. Diab and A. Makhlouf. This is an open access article distributed under the Creative Commons Attribution License, which permits unrestricted use, distribution, and reproduction in any medium, provided the original work is properly cited.

We perturb the differential system $\dot{x}_{1}=-x_{2}\left(1+x_{1}\right), \dot{x}_{2}=x_{1}\left(1+x_{1}\right)$, and $\dot{x}_{k}=0$ for $k=3, \ldots, d$ inside the class of all polynomial differential systems of degree $n$ in $\mathbb{R}^{d}$, and we prove that at most $n^{d-1}$ limit cycles can be obtained for the perturbed system using the first-order averaging theory.

\section{Introduction}

One of the main problems in the theory of differential systems is the study of their periodic orbits, their existence, their number, and their stability. As usual, a limit cycle of a differential system is a periodic orbit isolated in the set of all periodic orbits of the differential system.

In [1], the authors studied the differential system

$$
\begin{aligned}
& \dot{x}=-y+\varepsilon(a x+P(x, y)), \\
& \dot{y}=x+\varepsilon(a y+Q(x, y)),
\end{aligned}
$$

where $P(x, y)$ and $Q(x, y)$ are arbitrary polynomials of degree $n$ starting with terms of degree $2, a$ is a real parameter, and $\varepsilon$ is small parameter. They proved that for $\varepsilon \neq 0$ sufficiently small, the maximum number of limit cycles bifurcating from the periodic orbits of the linear center $\dot{x}=-y, \dot{y}=x$, obtained using the averaging theory of first order, is $(n-1) / 2$ if $n$ is odd and $(n-2) / 2$ if $n$ is even. In the same paper, the authors studied the limit cycles of the differential system

$$
\begin{aligned}
& \dot{x}=-y+\varepsilon(a x+P(x, y, z)), \\
& \dot{y}=x+\varepsilon(a y+Q(x, y, z)), \\
& \dot{z}=\varepsilon(b z+R(x, y, z)),
\end{aligned}
$$

where $P(x, y, z), Q(x, y, z)$, and $R(x, y, z)$ are arbitrary polynomials of degree $n$ starting with terms of degree 2 and $a, b \in$ $\mathbb{R}$. Then, there exists $\varepsilon_{0}>0$ sufficiently small such that for $|\varepsilon|<\varepsilon_{0}$ there are systems (2) having at least $n(n-1) / 2$ limit cycles bifurcating from the periodic orbits of the system $\dot{x}=-y, \dot{y}=x$, and $\dot{z}=0$.

In [2], the authors studied the number of limit cycles of the differential system

$$
\begin{aligned}
& \dot{x}=-y(1+x)+\varepsilon(a x+P(x, y, z)), \\
& \dot{y}=x(1+x)+\varepsilon(a y+Q(x, y, z)), \\
& \dot{z}=\varepsilon(b z+R(x, y, z)),
\end{aligned}
$$

where $F(x, y, z), G(x, y, z)$, and $R(x, y, z)$ are polynomials of degree $n$ starting from terms of degree 2 . Then, there exists $\varepsilon_{0}>0$ sufficiently small such that for $|\varepsilon|<\varepsilon_{0}$ there are systems (3) having at least $n^{2}$ limit cycles bifurcating from the periodic orbits of the system $\dot{x}=-y(1+x), \dot{y}=x(1+x)$, and $\dot{z}=0$.

In general, to obtain analytically periodic solutions of a differential system is a very difficult work, usually impossible. 
Here, using the averaging theory of first order, we will study the number of limit cycles of the differential system

$$
\begin{aligned}
& \dot{x}_{1}=-x_{2}\left(1+x_{1}\right)+\varepsilon\left(a x_{1}+P_{1}\left(x_{1}, \ldots, x_{d}\right)\right), \\
& \dot{x}_{2}=x_{1}\left(1+x_{1}\right)+\varepsilon\left(a x_{2}+P_{2}\left(x_{1}, \ldots, x_{d}\right)\right), \\
& \dot{x}_{k}=\varepsilon\left(b_{k} x_{k}+P_{k}\left(x_{1}, \ldots, x_{d}\right)\right), \quad k=3, \ldots, d,
\end{aligned}
$$

in $\mathbb{R}^{d}$, where $P_{k}\left(x_{1}, \ldots, x_{d}\right)$ for $k=1, \ldots, d$ is a polynomial of degree $n$ starting with terms of degree $2, a, b_{k} \in \mathbb{R}$, and $\varepsilon$ is a small parameter.

The problem of studying the limit cycles of system (4) is reduced using the averaging theory of first order to find the zeros of a nonlinear system of $d-2$ equations with $d-2$ unknowns. It is known that in general the averaging theory for finding periodic solutions does not provide all the periodic solutions of the system; this is due to two main reasons. First, the averaging theory for studying the periodic solutions of a differential system is based on the so-called displacement function, whose zeros provide periodic solutions of the differential system. This displacement function in general is not global and consequently it cannot control all the periodic solutions of the differential system, only the ones which are in its domain of definition and are hyperbolic. Second, the displacement function is expanded in power series of a small parameter $\varepsilon$, and the averaging theory only controls the zeros of the dominant term of this displacement function. When the dominant term is $\varepsilon^{k}$, we talk about the averaging theory of order $k$. For more details, see, for instance, [3] and the references quoted there. The averaging theory of first order necessary for the results of this paper is summarized in Section 2.

Our main result on the limit cycles of the differential system (4) is as follows.

Theorem 1. By applying the first-order averaging theory to the polynomial differential system (4), for $\varepsilon \neq 0$ sufficiently small at most $n^{d-1}$ limit cycles bifurcate from the periodic orbits of the differential system $\dot{x}_{1}=-x_{2}\left(1+x_{1}\right), \dot{x}_{2}=x_{1}\left(1+x_{1}\right), \dot{x}_{3}=0$, and $\dot{x}_{4}=0, \ldots, \dot{x}_{d}=0$.

Theorem 1 is proved in Section 3.

\section{Limit Cycles via Averaging Theory}

Roughly speaking, we can say that the averaging theory gives a quantitative relation between periodic solutions of a nonautonomous periodic differential system and the solutions of its averaged differential system, which is autonomous. The next result provides a first-order approximation in $\varepsilon$ for the limit cycles of a periodic differential system; for a proof, see Theorem 2.6.1 of [4] and Theorem 11.5 of [5].

Theorem 2. One considers the following two initial-value problems:

$$
\begin{aligned}
& \dot{x}=\varepsilon f(t, x)+\varepsilon^{2} g(t, x, \varepsilon), \quad x(0)=x_{0}, \\
& \dot{y}=\varepsilon f_{0}(y), \quad y(0)=x_{0},
\end{aligned}
$$

where $x, y, x_{0} \in D$, and $D$ is an open subset of $\mathbb{R}^{n}, t \in[0, \infty)$, $|\varepsilon| \leq \varepsilon_{0}, t \in[0, \infty),|\varepsilon| \leq \varepsilon_{0}, f$ and $g$ are periodic of period $T$ in the variable $t$, and $f_{0}(y)$ is the averaged function of $f(t, x)$ with respect to $t$; that is,

$$
f_{0}(y)=\frac{1}{T} \int_{0}^{T} f(t, y) d t
$$

Assume that

(i) $f$, its Jacobian $\partial f / \partial x$, its Hessian $\partial^{2} f / \partial x^{2}, g$, and its Jacobian $\partial g / \partial x$ are defined, continuous, and bounded by a constant independent of $\varepsilon$ in $[0, \infty) \times D$ and $|\varepsilon| \leq$ $\varepsilon_{0}$;

(ii) $T$ is a constant independent of $|\mathcal{\varepsilon}|$;

(iii) $y(t)$ belongs to $D$ on the time interval $[0,1 /|\varepsilon|]$.

Then, the following statements hold:

(a) On the time scale $1 /|\varepsilon|$, we have that $x(t)-y(t)=O(\varepsilon)$ as $\varepsilon \rightarrow 0$.

(b) If $p$ is a singular point of the averaged system (6) such that the determinant of the Jacobian matrix $\partial f_{0} /\left.\partial y\right|_{y=p}$ is not zero, then there exists a limit cycle $\varphi(t, \varepsilon)$ of period $T$ for system (5) such that $\varphi(0, \varepsilon) \rightarrow p$ as $\varepsilon \rightarrow 0$.

(c) The stability or instability of the limit cycle $\varphi(t, \varepsilon)$ is given by the stability or instability of the singular point $p$ of the averaged system (6) when $p$ is a hyperbolic singular point.

To prove Theorem 1, we need the following three lemmas which are proved in [6].

Before doing the proof of Theorem 1, we recall the Bézout theorem which will be used later on; for a proof of this result, see [7].

Theorem 3 (Bézout theorem). Let $q_{j}$ be polynomials in the variables $\left(x_{1}, \ldots, x_{d}\right)$ of degree $d_{j}$ for $j=1, \ldots, d$. Consider the following polynomial system: $q_{1}\left(x_{1}, \ldots, x_{d}\right)=$ $0, \ldots, q_{d}\left(x_{1}, \ldots, x_{d}\right)=0$, where $\left(x_{1}, \ldots, x_{d}\right) \in \mathbb{R}^{d}$. If the number of solutions of this system is finite, then it is bounded by $d_{1} \cdots d_{d}$.

Lemma 4. For $i, j \in \mathbb{N}$, one defines

$$
I_{i, j}=\frac{1}{2 \pi} \int_{0}^{2 \pi} \frac{\cos ^{i} \theta \sin ^{j} \theta}{1+\cos \theta} d \theta .
$$

Then, $I_{i, j}=0$ if and only if $j$ is even. For $i, j \in \mathbb{N}$ with $j$ even, one has

$$
I_{i, j}=\sum_{\substack{s=0 \\
s=v e n}}^{j}(-1)^{s / 2}\left(\begin{array}{c}
\frac{j}{2} \\
\frac{s}{2}
\end{array}\right) I_{i+s, 0} .
$$


Lemma 5. The following equalities hold. For $k \in \mathbb{N}$, one has

$$
\begin{aligned}
& E_{k}=\frac{1}{2 \pi} \int_{0}^{2 \pi} \cos ^{k} \theta d \theta= \begin{cases}0 & \text { if } k \text { is odd }, \\
C_{k}^{k / 2} 2^{-k} & \text { if } k \text { is even; }\end{cases} \\
& I_{0,0}=\frac{1}{2 \pi} \int_{0}^{2 \pi} \frac{1}{1+r \cos \theta} d \theta=\frac{1}{\sqrt{1-r^{2}}} .
\end{aligned}
$$

Lemma 6. For $i \in \mathbb{N}$, one has

$$
\begin{aligned}
I_{i, 0} & =\frac{1}{2 \pi} \int_{0}^{2 \pi} \frac{\cos ^{i} \theta}{1+r \cos \theta} d \theta \\
& =\frac{(-1)^{i}}{r^{i} \sqrt{1-r^{2}}}+\sum_{\substack{l=1 \\
l \equiv i(\bmod 2)}}^{i}(-1)^{l-1} 2^{l-i}\left(\begin{array}{c}
i-l \\
\frac{(i-l)}{2}
\end{array}\right) r^{-l} .
\end{aligned}
$$

\section{Proof of Theorem 1}

Doing the change to polar coordinates $x_{1}=r \cos \theta, x_{2}=$ $r \sin \theta$, system (4) becomes

$$
\begin{aligned}
\dot{r}= & \varepsilon\left(a r+\sum_{i=2}^{n} \sum_{i_{1}+\cdots+i_{d}=i} r^{i_{1}+i_{2}} x_{3}^{i_{3}}\right. \\
& \left.\cdots x_{d}^{i_{d}}\left(a_{i_{1}, i_{2}, \ldots, i_{d}}^{i_{1}} \cos ^{i_{1}+1} \theta \sin ^{i_{2}} \theta+a_{i_{1}, i_{2}, \ldots, i_{d}}^{i_{2}} \cos ^{i_{1}} \theta \sin ^{i_{2}+1} \theta\right)\right) \\
\dot{\theta}= & 1+r \cos \theta+\frac{\varepsilon}{r}\left(\sum_{i=2}^{n} \sum_{i_{1}+\cdots+i_{d}=i} r^{i_{1}+i_{2}} x_{3}^{i_{3}}\right. \\
& \left.\cdots x_{d}^{i_{d}}\left(a_{i_{1}, i_{2}, \ldots, i_{d}}^{i_{2}} \cos ^{i_{1}+1} \theta \sin ^{i_{2}} \theta-a_{i_{1}, i_{2}, \ldots, i_{d}}^{i_{1}} \cos ^{i_{1}} \theta \sin ^{i_{2}+1} \theta\right)\right) \\
\dot{x}_{k}= & \varepsilon\left(b_{k} x_{k}+\sum_{i=2}^{n} \sum_{i_{1}+\cdots+i_{d}=i} a_{i_{1}, i_{2}, \ldots, i_{d}}^{i^{i_{1}}} r^{i_{1}+i_{2}} x_{3}^{i_{3}} \cdots x_{d}^{i_{d}} \cos ^{i_{1}} \theta\right. \\
& \cdot \sin ^{\left.i_{2} \theta\right)},
\end{aligned}
$$

where $k=3, \ldots, d$. Taking $\theta$ as the new independent variable instead of $t$, this differential system can be written as

$$
\begin{aligned}
\frac{d r}{d \theta} & =\varepsilon F\left(\theta, r, x_{3}, \ldots, x_{d}\right)+O\left(\varepsilon^{2}\right), \\
\frac{d x_{k}}{d \theta} & =\varepsilon\left(G_{k}\left(\theta, r, x_{3}, \ldots, x_{d}\right)\right)+O\left(\varepsilon^{2}\right),
\end{aligned}
$$

for $k=3, \ldots, d$, where

$$
\begin{aligned}
& F\left(\theta, r, x_{3}, \ldots, x_{d}\right)=\operatorname{ar} D_{0,0}+\sum_{i=2}^{n} \sum_{i_{1}+\cdots+i_{d}=i} r^{i_{1}+i_{2}} x_{3}^{i_{3}} \\
& \quad \ldots x_{d}^{i_{d}}\left(a_{i_{1}, i_{2}, \ldots, i_{d}}^{i, 1} D_{i_{1}+1, i_{2}}+a_{i_{1}, i_{2}, \ldots, i_{d}}^{i, 2} D_{i_{1}, i_{2}+1}\right), \\
& G_{k}\left(\theta, r, x_{3}, \ldots, x_{d}\right)=b_{k} x_{d} D_{0,0} \\
& +\sum_{i=2}^{n} \sum_{i_{1}+\cdots+i_{d}=i} a_{i_{1}, i_{2}, \ldots, i_{d}}^{i_{1}} r^{i_{1}+i_{2}} x_{3}^{i_{3}} \ldots x_{d}^{i_{d}} D_{i_{1}, i_{2}},
\end{aligned}
$$

with

$$
D_{i_{1}, i_{2}}=\frac{\cos ^{i_{1}} \theta \sin ^{i_{2}} \theta}{1+r \cos \theta} .
$$

Now, using the notation introduced in Lemma 4 and applying the first-order averaging method, we must find the zeros of the system

$$
\begin{aligned}
f\left(r, x_{3}, \ldots, x_{d}\right) & =0, \\
g_{k}\left(r, x_{3}, \ldots, x_{d}\right) & =0, \text { for } k=3, \ldots, d,
\end{aligned}
$$

where

$$
\begin{aligned}
& f\left(r, x_{3}, \ldots, x_{d}\right)=\frac{1}{2 \pi} \int_{0}^{2 \pi} F\left(\theta, r, x_{3}, \ldots, x_{d}\right) d \theta \\
& =a r I_{0,0}+\sum_{i=2}^{n} \sum_{i_{1}+\cdots+i_{d}=i} r^{i_{1}+i_{2}} x_{3}^{i_{3}} \\
& \quad \ldots x_{d}^{i_{d}}\left(a_{i_{1}, i_{2}, \ldots, i_{d}}^{i, 1} I_{i_{1}+1, i_{2}}+a_{i_{1}, i_{2}, \ldots, i_{d}}^{i_{2}} I_{i_{1}, i_{2}+1}\right), \\
& g_{k}\left(r, x_{3}, \ldots, x_{d}\right)=\frac{1}{2 \pi} \int_{0}^{2 \pi} G_{k}\left(\theta, r, x_{3}, \ldots, x_{d}\right) d \theta \\
& =b_{k} x_{k} I_{0,0}+\sum_{i=2}^{n} \sum_{i_{1}+\cdots+i_{d}=i} a_{i_{1}, i_{2}, \ldots, i_{d}}^{i_{1}} r^{i_{1}+i_{2}} x_{3}^{i_{3}} \cdots x_{d}^{i_{d}} I_{i_{1}, i_{2}},
\end{aligned}
$$

for $k=3, \ldots, d$, and

$$
I_{i, j}=\frac{1}{2 \pi} \int_{0}^{2 \pi} D_{i_{1}, i_{2}} d \theta=\frac{1}{2 \pi} \int_{0}^{2 \pi} \frac{\cos ^{i_{1}} \theta \sin ^{i_{2}} \theta}{1+\cos \theta} d \theta .
$$

Theorem 7. Let $t=\sqrt{1-r^{2}}$ and $k=3, \ldots, d$. The function $\operatorname{tg}_{k}\left(t, x_{3}, \ldots, x_{d}\right)$ is a polynomial of degree $n$ in the variables $t$ and $x_{k}$, while $r t f\left(t, x_{3}, \ldots, x_{d}\right)$ is a polynomial of degree $n+1$. Moreover, $r t f\left(t, x_{3}, \ldots, x_{d}\right)=(t-1) Q\left(t, x_{3}, \ldots, x_{d}\right)$, where $Q$ is a polynomial in the variables $t$ and $x_{3}, x_{4}, \ldots, x_{d}$ of the degree at most $n$.

Proof. The function $g_{k}$ is a linear combination of $x_{k} I_{0,0}$ and $r^{i_{1}+i_{2}} x_{3}^{i_{3}} \cdots x_{d}^{i_{d}} I_{i_{1}, i_{2}}$, where $2 \leq i_{1}+i_{2}+\cdots+i_{d} \leq n$.

Lemma 4 claims that

$$
r^{m} I_{m, 0}=(-1)^{m}\left(\sqrt{1-r^{2}}\right)^{-1}+X_{m}(r),
$$

where $X_{m}$ is an even polynomial of the degree $m-1$ if $m$ is odd and of degree $m-2$ otherwise. Using the variable $t=\sqrt{1-r^{2}}$, we conclude that

$$
r^{m} I_{m, 0}=\frac{(-1)^{m}+\widehat{X}_{m}(t)}{t},
$$

where $\widehat{X}_{m}(t)$ is an odd polynomial of degree $m$ or $m-1$. Since $r^{m} I_{m, 0}$ vanishes at $r=0$, the functions $r^{k} I_{k, 0}$ for $k=2, \ldots, m$ span the space of functions of the form $[A+\widehat{X}(t)] / t$ vanishing at $t=1$ with $\operatorname{deg} \widehat{X}(t)=m$ or $m-1$, respectively. Lemma 4 implies that any function $r^{i+j} I_{i, j}$ is of the form

$$
\phi_{i, j}=\frac{Y_{i, j}(t)+\widehat{X}_{i+j}(t)}{t},
$$


where $Y_{i, j}(t)$ is an even polynomial in $t$ of the degree $j$ ( $j$ is necessarily even by Lemma 4$)$ and $\widehat{X}_{i+j}(t)$ is a polynomial in $t$ of the degree $i+j$ or $i+j-1$. We conclude that the functions $r^{i_{1}+i_{2}} I_{i_{1}, i_{2}}$, where $2-\left(i_{3}+\cdots+i_{d}\right) \leq i_{1}+i_{2} \leq$ $n-\left(i_{3}+\cdots+i_{d}\right)$, generate the space of functions $Z(t) / t$, where $\operatorname{deg} Z \leq n-\left(i_{3}+\cdots+i_{d}\right)$ (and, in addition, $\left.Z(1)=0\right)$. Therefore, $\left\{P_{k}\left(t, x_{3}, \ldots, x_{d}\right) / t, \operatorname{deg} P_{k} \leq n\right\}, k=3, \ldots, d$.

In a similar way, $f$ is a linear combination of $r$ and terms $r^{i_{1}+i_{2}} x_{3}^{i_{3}} \cdots x_{d}^{i_{d}} I_{i_{1}+1, i_{2}}$ and $r^{i_{1}+i_{2}} x_{3}^{i_{3}} \cdots x_{d}^{i_{d}} I_{i_{1}, i_{2}+1}$, where $2 \leq i_{1}+$ $i_{2}+\cdots+i_{d} \leq n$. We conclude that the functions $r^{i_{1}+i_{2}} I_{i_{1}+1, i_{2}}$ and $r^{i_{1}+i_{2}} I_{i_{1}, i_{2}+1}$, where $2-\left(i_{3}+\cdots+i_{d}\right) \leq i_{1}+i_{2} \leq n-\left(i_{3}+\cdots+i_{d}\right)$, generate the space of functions $Z(t) / r t$, where $\operatorname{deg} Z \leq n+$ $1-\left(i_{3}+\cdots+i_{d}\right)$. We have $f\left(0, x_{3}, \ldots, x_{d}\right)=0$ which implies $Z(1)=0$. Therefore, $\left\{(t-1) Q\left(t, x_{3}, \ldots, x_{d}\right) / r t, \operatorname{deg} Q \leq n\right\}$. So the polynomials $Q\left(t, x_{3}, \ldots, x_{d}\right)$ and $t g_{k}\left(t, x_{3}, \ldots, x_{d}\right)$ in the variables $t, x_{3}, \ldots, x_{d}$ have at most degree $n$. Hence, by the Bézout theorem, the maximum number of solutions of $\operatorname{tg}_{k}\left(t, x_{3}, \ldots, x_{d}\right)=0$ for $k=3, \ldots, d$ and $Q\left(t, x_{3}, \ldots, x_{d}\right)=0$ is at most $n^{d-1}$ for $0<t<1$.

Thus, from Theorems 2 and 3, it follows that the maximum number of limit cycles bifurcating from the differential system (4) is $n^{d-1}$ obtained using the averaging theory of first order. This completes the proof of Theorem 1 .

\section{An Application of Theorem 1}

In system (4), we consider the case $n$ even and

$$
\begin{aligned}
& P_{1}\left(x_{1}, \ldots, x_{d}\right)=\sum_{i=2}^{n} a_{0,0, \ldots, i}^{i, 1} x_{d}^{i}+a_{1,0, \ldots, 0,1}^{2,1} x_{1} x_{d}, \\
& P_{2}\left(x_{1}, \ldots, x_{d}\right)=0 \\
& P_{k}\left(x_{1}, \ldots, x_{d}\right)=\sum_{i=2, i \text { even }}^{n}\left(a_{i, 0, \ldots, 0}^{i, k} x_{1}^{i}+a_{0, i, \ldots, 0}^{i, k} x_{2}^{i}\right)
\end{aligned}
$$

Computing the averaged functions and taking $t=\sqrt{1-r^{2}}$, we have

$$
\begin{aligned}
r \sqrt{1-r^{2}} f\left(r, x_{3}, x_{4}, \ldots, x_{d}\right) \\
=a r^{2} \\
\quad+\left(a_{1,0, \ldots, 0,1}^{2,1} x_{d}-\sum_{i=2}^{n} a_{0,0, \ldots, i}^{i, 1} x_{d}^{i}\right)\left(1-\sqrt{1-r^{2}}\right) \\
=(1-t)\left(a(1+t)+a_{1,0, \ldots, 0,1}^{2,1} x_{d}-\sum_{i=2}^{n} a_{0,0, \ldots, i}^{i, 1} x_{d}^{i}\right) \\
=(1-t)\left(a(1+t)-\bar{Q}\left(x_{d}\right)\right),
\end{aligned}
$$

where $\bar{Q}\left(x_{d}\right)$ is an arbitrary polynomial in $x_{d}$ of degree $n$ such that $\bar{Q}(0)=0$. At the same time, the averaged function corresponding to $P_{k}\left(x_{1}, \ldots, x_{d}\right)$ satisfies

$$
\begin{aligned}
& \sqrt{1-r^{2}} g_{k}\left(r, x_{3}, x_{4}, \ldots, x_{d}\right) \\
& =b_{k} x_{k}+\sqrt{1-r^{2}} \sum_{\substack{i=2 \\
i \text { even }}}^{n} r^{i}\left(a_{i, 0,0, \ldots, 0,0}^{i, k} I_{i, 0}+a_{0, i, 0, \ldots, 0}^{i, k} I_{0, i}\right),
\end{aligned}
$$

for $k=3, \ldots, d$. It is easy to obtain the following relations:

$$
\begin{aligned}
& \frac{r^{k} \cos ^{k} \theta}{1+r \cos \theta}=(-1)^{k} \frac{1}{1+r \cos \theta} \\
& +\sum_{v=1}^{k}(-1)^{k} \cos ^{k-v} \theta r^{k-v}, \\
& \frac{r^{k} \sin ^{k} \theta}{1+r \cos \theta}=\sum_{s=0, s \text { even }}^{k}(-1)^{s / 2}\left(\begin{array}{c}
\frac{k}{2} \\
\frac{s}{2}
\end{array}\right) \\
& \cdot\left[(-1)^{k} \frac{r^{k-s}}{1+r \cos \theta}+\sum_{v=1}^{s}(-1)^{v-1} \cos ^{s-v} \theta r^{k-v}\right] .
\end{aligned}
$$

Looking at the second term of the first relation and at the first term of the second relation, we obtain that $r^{i} I_{i, 0}$ and $r^{i} I_{0, i}$ for even $2 \leq i \leq n$ are independent. In particular, using Lemmas 4,5 , and 6 , we obtain

$$
\begin{aligned}
& \sqrt{1-r^{2}} g_{k}\left(r, x_{3}, x_{4}, \ldots, x_{d}\right) \\
& =b_{k} x_{k}+g_{1, k}(r)+g_{2, k}(r), \quad \text { for } k=3, \ldots, d,
\end{aligned}
$$

where

$$
g_{1, k}=\sum_{\substack{i=2 \\ i \text { even }}}^{n} a_{i, 0,0, \ldots, 0}^{i, k}
$$

$$
\begin{array}{r}
-\sqrt{1-r^{2}} \sum_{\substack{m=0 \\
m \text { even }}}^{n-1} r^{m} \sum_{\substack{i=m+1 \\
i \text { even }}}^{n} a_{i, 0, \ldots, 0}^{i, k} 2^{-m}\left(\begin{array}{c}
m \\
\frac{m}{2}
\end{array}\right), \\
g_{2, k}=\sum_{\substack{m=0 \\
m \text { even }}}^{n} A_{m, k} r^{m}+\sqrt{1-r^{2}} \sum_{\substack{m=0 \\
m \text { even }}}^{n-1} B_{m, k} r^{m},
\end{array}
$$

where

$$
\begin{aligned}
A_{m, k} & =\sum_{\substack{i=0 \\
i \text { even }}}^{n} a_{0, i, 0, \ldots, 0}^{i, k} d_{i-m, i}, \\
B_{m, k} & =\sum_{\substack{i=0 \\
i \text { even }}}^{n} a_{0, i, 0, \ldots, 0}^{i, k} d_{i-m, i} \sum_{\substack{l>0 \\
l \text { even }}}^{n} e_{i-m, l},
\end{aligned}
$$




$$
\begin{aligned}
& d_{i-m, i}=(-1)^{(i-m) / 2}\left(\begin{array}{c}
\frac{i}{2} \\
\frac{(i-m)}{2}
\end{array}\right), \\
& e_{i-m, l}=(-1)^{l-1} 2^{l-(i-m)}\left(\begin{array}{c}
i-m-l \\
\frac{(i-m-l)}{2}
\end{array}\right) .
\end{aligned}
$$

Writing $t=\sqrt{1-r^{2}}$, the polynomials $g_{s, k}(r)=P_{s, k}(t)$ satisfy the conditions $g_{s, k}(0)=P_{s, k}(1)=0$ for $s=1,2$ and $k=$ $3, \ldots, d$. Then, we can define a polynomial of degree $n$ in $t$ :

$$
\bar{P}_{k}(t)=P_{1, k}(t)+P_{2, k}(t)=(t-1) \widetilde{P}_{k}(t) .
$$

Due to the independence of $r^{i} I_{i, 0}$ and $r^{i} I_{0, i}$ and the arbitrariness of the coefficients $a_{i, 0,0, \ldots, 0}^{i, k}$ and $a_{0, i, 0, \ldots, 0}^{i, k}$, the polynomial $\bar{P}_{k}(t)$ is an arbitrary polynomial such that $\bar{P}_{k}(1)=0$. In fact, it is obvious that $g_{1, k}$ and $g_{2, k}$ have $n / 2$ parameters, respectively, where $n / 2$ coefficients $a_{0, i, 0, \ldots, 0}^{i, k}$ allow choosing the first term of $g_{2, k}$ arbitrarily except for the term with $m=0$, implying that the even terms of $\overline{P_{k}}(t)$ are arbitrary except for the constant term, while the other $n / 2$ coefficients $a_{i, 0,0, \ldots, 0}^{i, k}$ allow choosing the second term in $g_{1, k}$ arbitrarily, implying that the odd terms of $\overline{P_{k}}(t)$ are arbitrary. Therefore, the polynomial $\overline{P_{k}}(t)$ of the degree $n$ satisfies $\overline{P_{k}}(1)=0$ and has $n$ arbitrary coefficients. The number of solutions of $f\left(r, x_{3}, x_{4}, \ldots, x_{d}\right)=$ $0, g_{k}\left(r, x_{3}, x_{4}, \ldots, x_{d}\right)=0$, for $k=3, \ldots, d$, is equal to the number of the intersection points of the curves

$$
\begin{aligned}
l_{1}: a(1+t)-\bar{Q}\left(x_{d}\right) & =0 . \\
l_{k-1}: b_{k} x_{k}+\bar{P}_{k}(t) & =0, \text { for } k=3, \ldots, d .
\end{aligned}
$$

Hence, by the Bézout theorem, the maximum number of the common solutions of system (30) is at most $n^{d-1}$ for $0<t<1$. We can find $n^{d-1}$ intersection points on $f\left(r, x_{3}, \ldots, x_{d}\right)=0$ with $g_{k}\left(r, x_{3}, \ldots, x_{d}\right)=0$ for $k=3, \ldots, d, r \in\left(r_{0}, 1\right), 0<$ $r_{0} \ll 1$, which (using the averaging theory; see Theorem 2) give rise to $n^{d-1}$ limit cycles bifurcating from periodic orbits of the system $\dot{x}_{1}=-x_{2}\left(1+x_{1}\right), \dot{x}_{2}=x_{1}\left(1+x_{1}\right), \dot{x}_{3}=0$, and $\dot{x}_{4}=0, \ldots, \dot{x}_{d}=0$.

\section{Conflict of Interests}

The authors declare that there is no conflict of interests regarding the publication of this paper.

\section{References}

[1] A. Cima, J. Llibre, and M. A. Teixeira, "Limit cycles of some polynomial differential systems in dimension 2,3 and 4, via averaging theory," Applicable Analysis, vol. 87, no. 2, pp. 149-164, 2008.

[2] J. Llibre and J. Yu, "Limit cycles for a class of three-dimensional polynomial differential systems," Journal of Dynamical and Control Systems, vol. 13, no. 4, pp. 531-539, 2007.
[3] J. Llibre, D. D. Novaes, and M. A. Teixeira, "Higher order averaging theory for finding periodic solutions via Brouwer degree," Nonlinearity, vol. 27, no. 3, pp. 563-583, 2014.

[4] J. A. Sanders and F. Verhulst, Averaging Methods in Nonlinear Dynamical Systems, vol. 59, Springer, New York, NY, USA, 1985.

[5] F. Verhulst, Nonlinear Differential Equations and Dynamical Systems, Springer, Berlin, Germany, 1996.

[6] J. Llibre, J. S. Rio, and J. A. Rodriguez, "Averaging analysis of a perturbed quadratic center," Nonlinear Analysis, vol. 46, no. 1, pp. 45-51, 2001.

[7] I. R. Shafarevich, Basic Algebraic Geometry, Springer, Berlin, Germany, 1974. 


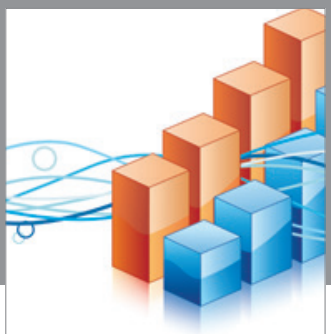

Advances in

Operations Research

vatem alat4

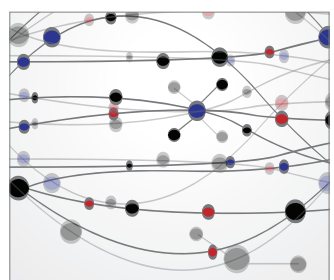

\section{The Scientific} World Journal
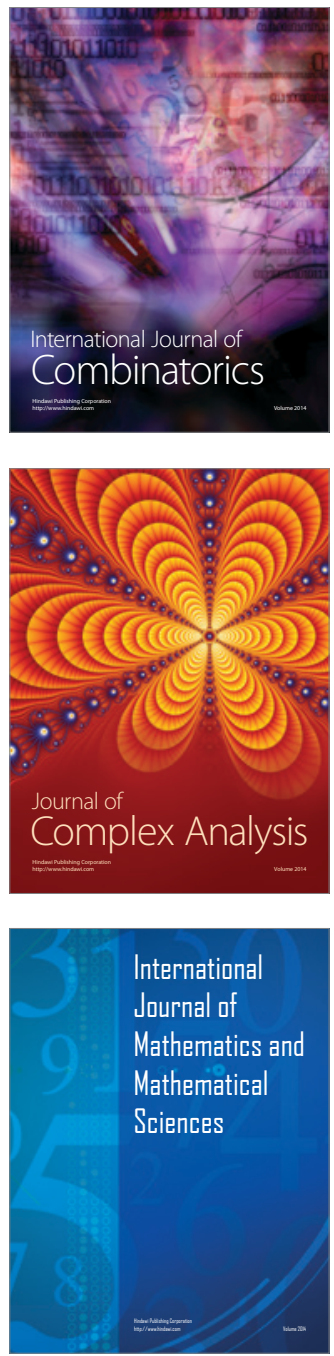
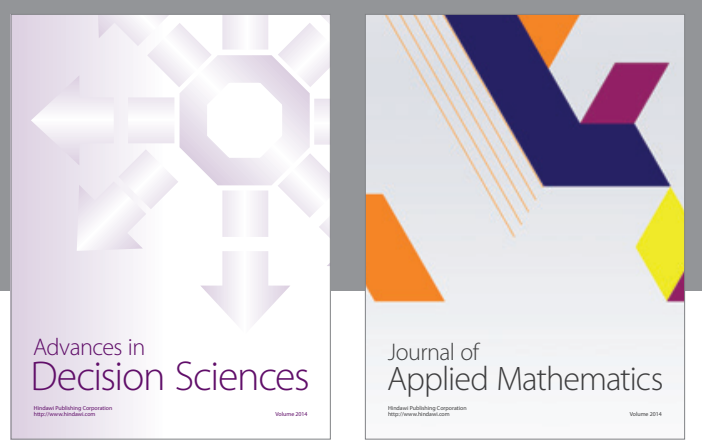

Algebra

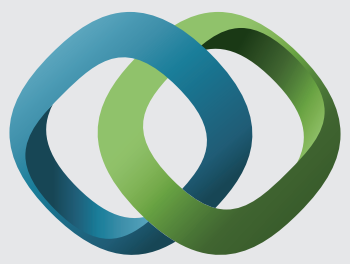

\section{Hindawi}

Submit your manuscripts at

http://www.hindawi.com
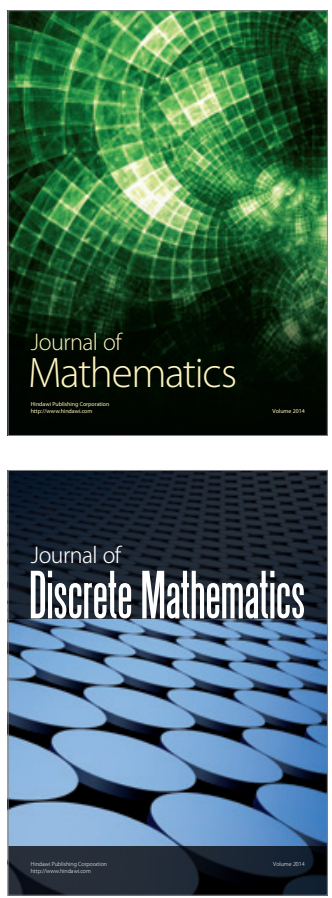

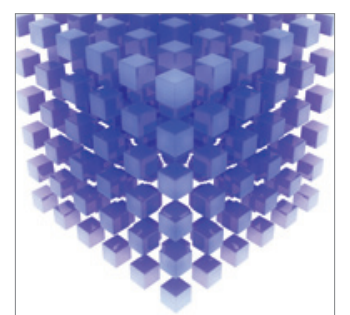

Mathematical Problems in Engineering
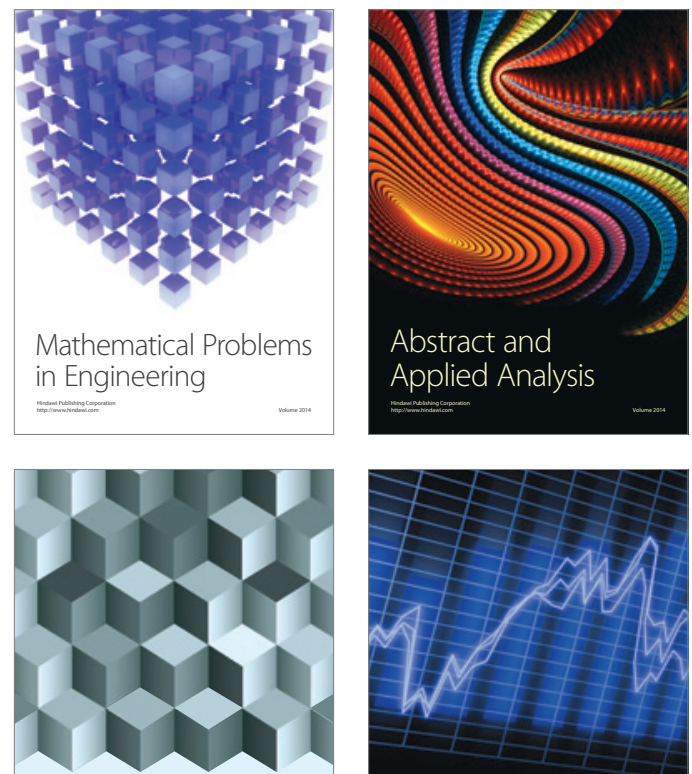

Journal of

Function Spaces

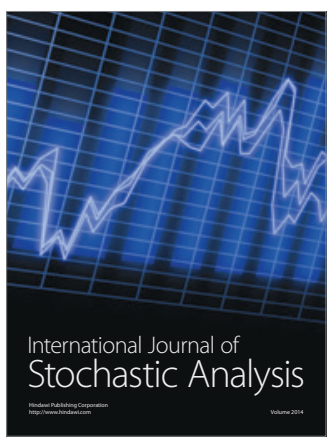

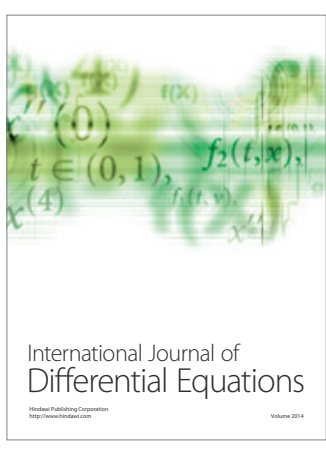
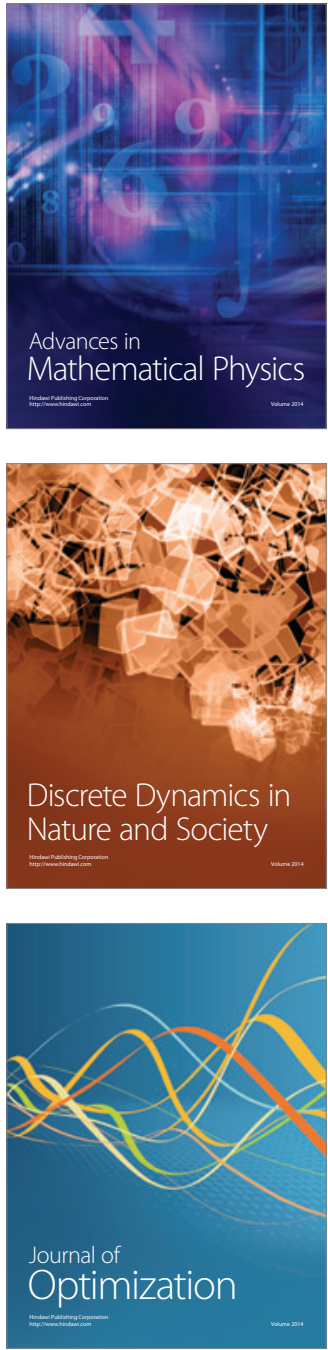